\title{
Compact and intense parametric $x$-ray radiation source based on a linear accelerator with cryogenic accelerating and decelerating copper structures
}

\author{
J. Hyun, ${ }^{1, *}$ M. Satoh,${ }^{1,2}$ M. Yoshida, ${ }^{1,2}$ T. Sakai, ${ }^{3}$ Y. Hayakawa, ${ }^{3}$ \\ T. Tanaka, ${ }^{3}$ K. Hayakawa, ${ }^{3}$ I. Sato, ${ }^{3}$ and K. Endo ${ }^{4}$ \\ ${ }^{1}$ SOKENDAI (The Graduate University for Advanced Studies), \\ 1-1 Oho, Tsukuba, Ibaraki 305-0801, Japan \\ ${ }^{2}$ High Energy Accelerator Research Organization (KEK), 1-1 Oho, Tsukuba, Ibaraki 305-0801, Japan \\ ${ }^{3}$ Laboratory for Electron Beam Research and Application (LEBRA), Nihon University, \\ 7-24-1, Narashinodai, Funabashi 274-8501, Japan \\ ${ }^{4}$ TOYAMA CO., Ltd. 3816-1 Kishi, Yamakita, Ashigarakami, Kanagawa 258-0112, Japan
}

(Received 6 February 2017; published 11 January 2018)

\begin{abstract}
This paper describes a proposal for a compact $\mathrm{x}$-ray source based on parametric $\mathrm{x}$-ray radiation (PXR). The PXR, which is produced when a single crystal is bombarded with relativistic electrons, has good monochromaticity and spatial coherence, and is expected to be well suited for imaging of low-Z materials and medical application. The proposed system employs a pair of copper accelerating structures which are operated at a cryogenic temperature of $20 \mathrm{~K}$ and arranged to form a resonant ring configuration. The electron beam is once accelerated up to $75 \mathrm{MeV}$ in one of the structures, being decelerated down to lower than $7 \mathrm{MeV}$ in the other structure after generating PXR at a single crystal, and then dumped. The expected $\mathrm{x}$-ray yield is $10^{9}$ photons/s at a center energy of $15 \mathrm{keV}$ or higher.
\end{abstract}

DOI: 10.1103/PhysRevAccelBeams.21.014701

\section{INTRODUCTION}

$\mathrm{X}$-rays have been utilized in a wide range of scientific fields as one of the essential tools for analysis and imaging including medical diagnosis since its discovery in 1895. Commonly used methods for producing $\mathrm{x}$-rays include: characteristic $\mathrm{x}$-rays and synchrotron radiation (SR) [1], each having different features of x-ray monochromaticity, wavelength tunability and intensity. The characteristic $\mathrm{x}$-rays are generated in an $\mathrm{x}$-ray tube by the transition of electrons from a higher atomic level to the vacant atomic level caused by energetic electrons incident on the target anode. While its energy can be chosen by selecting $\mathrm{Cu}(8.0 \mathrm{keV}), \mathrm{Mg}(1.2 \mathrm{keV})$ or Mo $(17.5 \mathrm{keV})$ [2] as the anode material, the spectrum of the X-rays for a given anode is monochromatic [3]. Its intensity depends on the accelerating voltage and the electron beam current, the photon flux being typically $10^{8}$ photons/s or lower. The SR, produced at electron storage rings, is emitted from the electrons passing through the bending field of dipole magnets. Its intensity is considerably higher, a typical photon flux at a user port being higher than $10^{9}$ photons/s when an

\footnotetext{
*hyon@post.kek.jp
}

Published by the American Physical Society under the terms of the Creative Commons Attribution 4.0 International license. Further distribution of this work must maintain attribution to the author(s) and the published article's title, journal citation, and DOI. electron beam of several hundred $\mathrm{mA}$ is accumulated in the storage ring. The spectrum of the SR is continuous, covering wavelengths from microwave to hard $\mathrm{x}$-rays, and the maximum energy of the x-rays depends on the electron beam energy. For example, to produce x-rays with an energy of $12 \mathrm{keV}$ for structural analyses, electrons with higher energies than $3 \mathrm{GeV}$ are required. To accelerate electrons up to a few $\mathrm{GeV}$, many accelerating structures are required. Moreover, a radiation shield with sufficient thickness is needed for radiation safety. Thus construction of a SR facility usually requires a large site footprint and high construction cost. From these considerations, a compact $\mathrm{x}$-ray source facility producing quasi-monochromatic $\mathrm{x}$-rays with a wide wavelength tunability is desired [4].

Parametric X-ray radiation (PXR) [5] is a radiation phenomenon induced by a relativistic charged particle passing through a single crystal when the Bragg diffraction condition is satisfied. The main advantage of PXR is that its energy is nearly independent of the energy of the incident particle. The energy of PXR depends on the Bragg angle and the crystal structure. For instance, PXR with an energy of about $8 \mathrm{keV}$ has been produced by using electron beams with energies below $10 \mathrm{MeV}[6,7]$. Thus, the required electron beam energy is considerably lower than what is required for SR. The energy spread of PXR is approximately 1\% [7]. It is much narrower than what is available from other $\mathrm{x}$-ray generation methods with a crystal and charged particles, for example, channeling radiation [8-10] and transition radiation [11]. The yield of PXR, which depends on a Fourier expansion of electric 
susceptibility, is at most $10^{-5}-10^{-6}$ photons/electron [12-14]. It is very low compared with SR. In order to increase the PXR intensity, it is necessary to bombard a crystal with a high average beam current.

Currently, x-ray imaging and XAFS (x-ray absorption fine structure) have been generally conducted with SR. A wealth of information on a crystal can be obtained by using XAFS measurement. XAFS, which requires a quasimonochromatic X-ray beam with wavelength tunability, can take advantage of PXR [15]. A group at Nihon University succeeded in obtaining the image of a low-Z material by phase contrast imaging with PXR at LEBRA (Laboratory for Electron Beam Research and Application, Nihon University) [16-18]. This result suggests that PXR has a spatial coherence and is suitable $\mathrm{X}$-ray source for $\mathrm{X}$-ray imaging. Moreover, efforts have been focused on obtaining a higher PXR intensity by using a crystal with an asymmetric cut surface to reduce X-ray absorption in the crystal. This has resulted in about five times higher than the PXR yield that was obtained by a crystal with a symmetric cut surface [19]. However, in order to be comparable to SR, the PXR intensity must be increased by another factor of a few hundred.

The development has been pursued to substantiate a compact, high intensity PXR source based on a linear accelerator which can be utilized for medical treatments, crystal structure analyses and imaging of biological cells. The target of the PXR yield is set higher than $10^{9}$ photons $/ \mathrm{s}$ at $15 \mathrm{keV}$ or higher, which is two orders of magnitude higher than with other PXR sources [16]. Estimation of the PXR generation using single crystals of $\mathrm{Si}$ and diamond in terms of the x-ray energy and yield suggests that an average beam power of $1.8 \mathrm{~kW}$ is required at an electron energy of $75 \mathrm{MeV}$. When electrons with this high power are dumped, a large and thick radiation shield must be constructed to ensure radiation safety. Thus, a decelerating structure is employed to dump the electrons at energies close to the injection energy. Moreover, we use a pair of the accelerator structures made of a high-pure copper (6N8), which is operated at a cryogenic temperature of $20 \mathrm{~K}$ to obtain a high Q-factor [20]. While superconducting cavities can achieve a high Q-factor of the order of $10^{8}-10^{10}$ at $1.5-4.2 \mathrm{~K}$ [21], they are not employed because of issues with quenches, field emission and increase in construction cost for cryogenics with a higher capability.

In Sec. II of this paper, the design concept of the compact intense PXR source is shown, where the details of the injector, the accelerating structure with cryogenic system and the $\mathrm{rf}$ resonant ring are described. The calculation result of neutron generation simulated with GEANT4 [22] is described in Sec. III. The simulation results of the expected PXR intensity in this X-ray source and the beam optics with and without emittance growth after a single crystal using SAD [23] and GEANT4 are shown in Sec. IV and V. The conclusions of the discussion on this PXR source are summarized in Sec. VI.

\section{CONCEPT OF PXR SOURCE}

In this section, the concept of the PXR source based on a linear accelerator is described. This PXR source is a demonstration machine possibly applicable to medical treatments, crystal structure analyses and imaging of biological organs/tissues in a medical facility or laboratory. Our goal on the photon flux is to achieve more than $10^{9}$ photons/s at over $15 \mathrm{keV}$. The beam power of about $1.8 \mathrm{~kW}$ at the beam energy of $75 \mathrm{MeV}$ is required to achieve this photon flux. This beam energy was chosen by considering a klystron power of $50 \mathrm{MW}$ currently available at the developing site, beam current $(<300 \mathrm{~mA})$ produced at an electron gun, and parameters of accelerating structures which can be developed, which is the minimum beam energy required to achieve the target photon yield. Moreover, the possibility of installation into a small clinical and laboratory site was taken into account. For a beam dump of the electron beam with that power, we must take a radiation shield for gamma rays and neutrons into account, and a thick block for the beam dump must be constructed. In order to realize low-level radiation at the beam dump, we employ a decelerating structure, also. The proposed PXR source is schematically shown in Fig. 1. Table I shows its main parameters.

The features of this PXR source are to reduce the generation of unwanted photons and particles such as neutrons, and accelerate a high current electron beam using copper accelerating structures operated at $20 \mathrm{~K}$. The footprint of this facility is 8 meters $\times 4$ meters and is substantially compact as compared with typical $100-\mathrm{MeV}$ class accelerators. The injector consists of a triode electron gun, an rf chopper, a prebuncher, a buncher, four quadrupole magnets, and accelerating and decelerating structures. The bunchers and accelerator structures are operated at the rf frequency of $5.712 \mathrm{GHz}$ with the repetition rate of $50 \mathrm{~Hz}$. The maximum rf output power and pulse length of the klystron are $50 \mathrm{MW}$ and $3.5 \mu \mathrm{s}$, respectively. The normalized emittance of the electron beam is expected to be

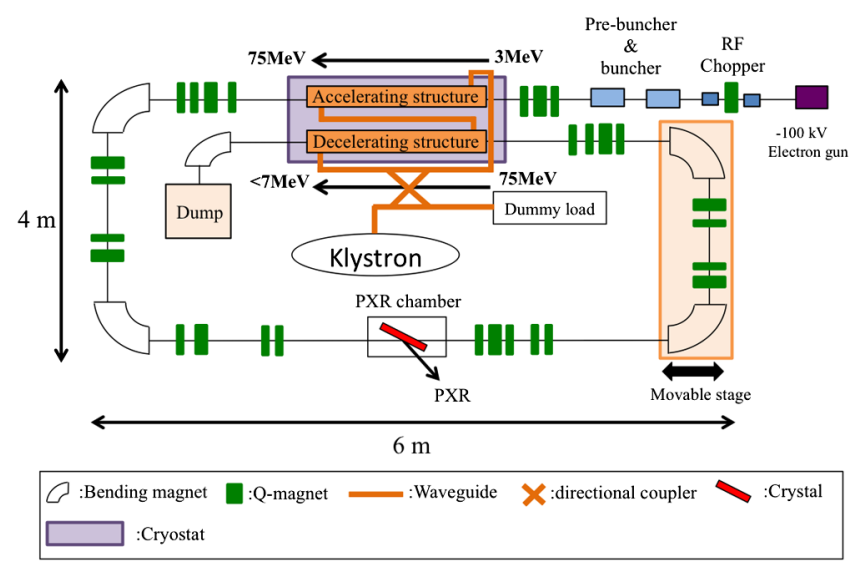

FIG. 1. Layout of PXR source based on linear accelerator. 
TABLE I. Basic parameters of the linac and two accelerating structures at $20 \mathrm{~K}$.

\begin{tabular}{lcc}
\hline \hline Parameters & Values & Units \\
\hline Beam energy & 75 & $\mathrm{MeV}$ \\
Peak current & $<300$ & $\mathrm{~mA}$ \\
Normalized emittance & 20 & $\mathrm{~mm}-\mathrm{mrad}$ \\
Acceleration frequency & 5712 & $\mathrm{MHz}$ \\
Pulse repetition rate & 50 & $\mathrm{~Hz}$ \\
rf pulse length & 3.5 & $\mu \mathrm{s}$ \\
Phase advance per cell & $2 \pi / 3$ & $\mathrm{rad}$ \\
Number of cells with couplers & 76 & \\
Iris diameter & $13.2-16$ & $\mathrm{~mm}$ \\
Disk spacing & 15 & $\mathrm{~mm}$ \\
Disk thickness & 2.5 & $\mathrm{~mm}$ \\
Accelerating structure length & 1.3 & $\mathrm{~mm}$ \\
\hline \hline
\end{tabular}

$20 \mathrm{~mm}$-mrad. The electrons produced at the electron gun have an energy of $3 \mathrm{MeV}$ at the exit of the buncher, and are accelerated up to $75 \mathrm{MeV}$ in the $1.3 \mathrm{~m}$ long accelerating structure. In the high energy beam transport line after the accelerating structure, four dipole magnets each having the bending angle of $90^{\circ}$ and the bend radius of $400 \mathrm{~mm}$ are used to bend the $75 \mathrm{MeV}$ electron beam, in which two dipole magnets (second arc section) after the crystal are placed on a movable stage to adjust the beam path length so that the electron bunchs are injected into the decelerating structure at a correct decelerating rf phase. The stage can slide up to $\pm 27 \mathrm{~mm}$, well over a full wavelength of the C-band rf.

This x-ray source is operated in a multibunch mode. One-turn operation is employed since the beam after passing through the crystal is not reusable due to the emittance growth. Before the beam is dumped, it is decelerated by a decelerating structure so as to reduce the radiation hazard as mentioned earlier. We adjust the longitudinal bunch length at the exit of the buncher by using an rf chopper [24] so that most of the electrons once accelerated to the maximum energy can be decelerated to $7 \mathrm{MeV}$ or lower in the decelerating structure. The accelerating and decelerating structures are contained in a cryostat and cooled conductively by connecting to the cold head of a GM (Gifford-McMahon) refrigerator. Moreover, the two structures are arranged to form a resonant ring configuration which allows us to obtain high gradient with the available rf power source, then the rf power dissipation in them will be a few $\mathrm{kW}$.

\section{ELECTRON ACCELERATOR}

An important consideration in designing a facility like this is to keep the radiation hazard under good control. Radiation dose outside the accelerator housing, whether due to photons, electrons or neutrons, is reduced by a combination of strategically arranged radiation shields and minimization of the beam loss within the accelerator, both

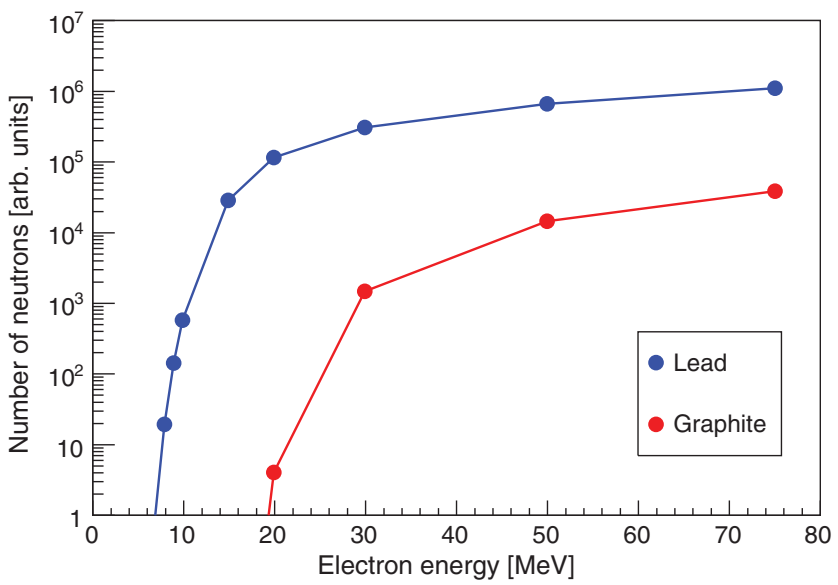

FIG. 2. Number of emitted neutrons dependence of electrons energy dumped at lead and graphite.

accidental and intentional. It is also noted that the production cross section of neutrons due to photonuclear reactions of electrons at the beam dump is highly sensitive to the incident electron energy and the mass number of the material exposed to the electrons. The number of the neutrons generated at the beam dump of lead and graphite has been estimated for different electron energies as shown in Fig. 2. This calculation was performed with GEANT4, a tool kit to simulate the interaction of particles and material [22], by assuming lead and graphite with a thickness of $20 \mathrm{~cm}$ and electrons of $10^{8}$ per pulse perpendicularly incident on it. Figure 2 indicates that production of neutrons is negligible when the electron bunch is dumped at the energy of $7 \mathrm{MeV}$ or below, as expected from the threshold energy of the photonuclear reaction of lead [25]. Therefore, dumping the electron beam after decelerated down to the energies less than $7 \mathrm{MeV}$ is preferable.

The longitudinal bunch length at the decelerating structure is important for ensuring the electron energy to be $7 \mathrm{MeV}$ or lower at the beam dump. The path length difference $\Delta z$ of an electron in a bending magnet is described by

$$
\Delta z=R_{56} \frac{\Delta P}{P},
$$

where $\Delta z$ is the path length difference of the electron with a momentum displacement $\Delta P / P$, and $R_{56}$ the momentum compaction $\left(R_{56}=\rho \theta-\rho \sin \theta\right)$ for the bending radius of $\rho$ and the bending angle of $\theta$. For the electron with a momentum offset of $\pm 0.25 \%$, the path length difference at the entrance of the decelerating structure will be

$\Delta z=4 \cdot\left(400 \cdot \frac{\pi}{2}-400 \cdot \sin \frac{\pi}{2}\right) \cdot \pm 0.0025= \pm 2.3 \mathrm{~mm}$,

which is considerably larger than the bunch length normally obtained at the exit of the buncher. The limit of the 
longitudinal bunch length required for the beam energy to be decelerated to $7 \mathrm{MeV}$ or lower at the beam dump can be calculated by the following equation with an energy gain and an rf phase

$$
E_{2}=E_{1}-E_{0} \cos \theta \Rightarrow \Delta E=-E_{0} \cos \theta,
$$

where $E_{2}$ is the electron energy at beam dump, $E_{1}$ the maximum beam energy, $E_{0}=e V_{0}, V_{0}$ the maximum decelerating voltage, $\Delta E\left(=E_{2}-E_{1}\right)$ the difference of energy and $\theta$ the phase of $\mathrm{rf}$ wave. If we choose $\Delta E=$ $68 \mathrm{MeV}$ at $E_{0}=72 \mathrm{MeV}$ as the worst case of deceleration corresponding to $E_{2}=7 \mathrm{MeV}$, the maximum allowed phase displacement of an electron from the bunch center adjusted to $\theta=0$ is

$$
\theta= \pm 19.2^{\circ} \rightarrow \Delta z= \pm 2.8 \mathrm{~mm}
$$

This suggests that the bunch length at the entrance of the decelerating structure must be less than $5.6 \mathrm{~mm}$. If the bunch length is $1 \mathrm{~mm}$ at the buncher exit, the bunch length at the decelerating structure entrance is about $5.6 \mathrm{~mm}$ $(= \pm 2.3 \mathrm{~mm}+1 \mathrm{~mm})$. In this case, the electron beam can be decelerated to $7 \mathrm{MeV}$ or lower. Therefore, the chopper is required at the buncher exit to limit the longitudinal beam size to be approximately $1 \mathrm{~mm}$ there $\left( \pm 3^{\circ}\right)$. We employ the rf chopper, which comprises two rf deflectors, a slit and a quadrupole magnet [24], use it to control bunch length in the low energy section $(100 \mathrm{keV})$ of the facility. The energy spread after passing through a crystal generally increases. However, the total electron energy decreases due to the energy loss. Therefore, requirement on the bunch length as examined above is expected to stay valid.

The accelerating and decelerating structures, are made of copper with 6N8 grade (purity $>99.999980$ mass\%), in the $2 \pi / 3$ traveling wave design. The two structure parameters are summarized in Table I. The iris with diameters of $13-16 \mathrm{~mm}$ is chosen to prevent particle loss in the structures, although the cell diameter should be small because of an increase of the shunt impedance. The cell's iris shape is ellipsoidal to reduce the peak surface electrical field as a discharge measure since high rf power is fed into the two structures. Also, they are $1.3 \mathrm{~m}$ long, and are operated at the temperature of $20 \mathrm{~K}$ for the long beam pulses [20]; the beam pulse and rf pulse length are approximately $2 \mu \mathrm{s}$ and $3 \mu \mathrm{s}$, respectively. In general, the surface resistance of copper is reduced when the structure's temperature is decreased, which results in an increase of the Q-factor. However, the surface resistance of a metal at a low temperature and high frequency of $\mathrm{GHz}$ is known to increase by the anomalous skin effect [26-29]. The surface resistance including the anomalous skin effect is

$$
\begin{gathered}
R_{s}=R_{\infty}\left(1+1.157 \alpha^{-0.276}\right), \\
R_{\infty}=1.123 \times 10^{-3} f^{2 / 3}, \quad \alpha=\frac{3}{4} \omega \mu_{0}(\rho \lambda)^{2} \rho^{-3},
\end{gathered}
$$

where $\omega$ is the angular frequency, $\mu_{0}$ the vacuum permeability, $\lambda$ the mean free path, $\rho$ the resistivity and $f$ the frequency in GHz. The residual resistivity ratio (RRR) of the 6N8 copper is expected to be 3,000 [30] or larger, and its surface resistance $R_{s}$ at $20 \mathrm{~K}$ is $\rho_{20 K} \sim 1.2 \times 10^{-11} \Omega \cdot \mathrm{m}$. Hence, the Q-factor and the shunt impedance at $20 \mathrm{~K}$ are expected to be approximately 50000 and $536 \mathrm{M} \Omega / \mathrm{m}$, respectively, which are about five times higher than those at a room temperature for the accelerator structure in our consideration.

To accelerate the beam up to $75 \mathrm{MeV}$, the accelerator structure has to operate at an accelerating gradient of $55 \mathrm{MV} / \mathrm{m}$, and a peak rf power of $140 \mathrm{MW}$ is required. The entire rf power in our system, including what is needed for the chopper, the prebuncher and the buncher, is supplied by a $50 \mathrm{MW}$ klystron. The rf power for accelerating structures is fed through the resonant ring that is arranged as shown by Figs. 3(a) and 3(b). The $a_{i}$ and $b_{i}(i=1,2,3$, 4) in Fig. 3(b) represent the incident waves and the outgoing waves. They satisfy the following condition,
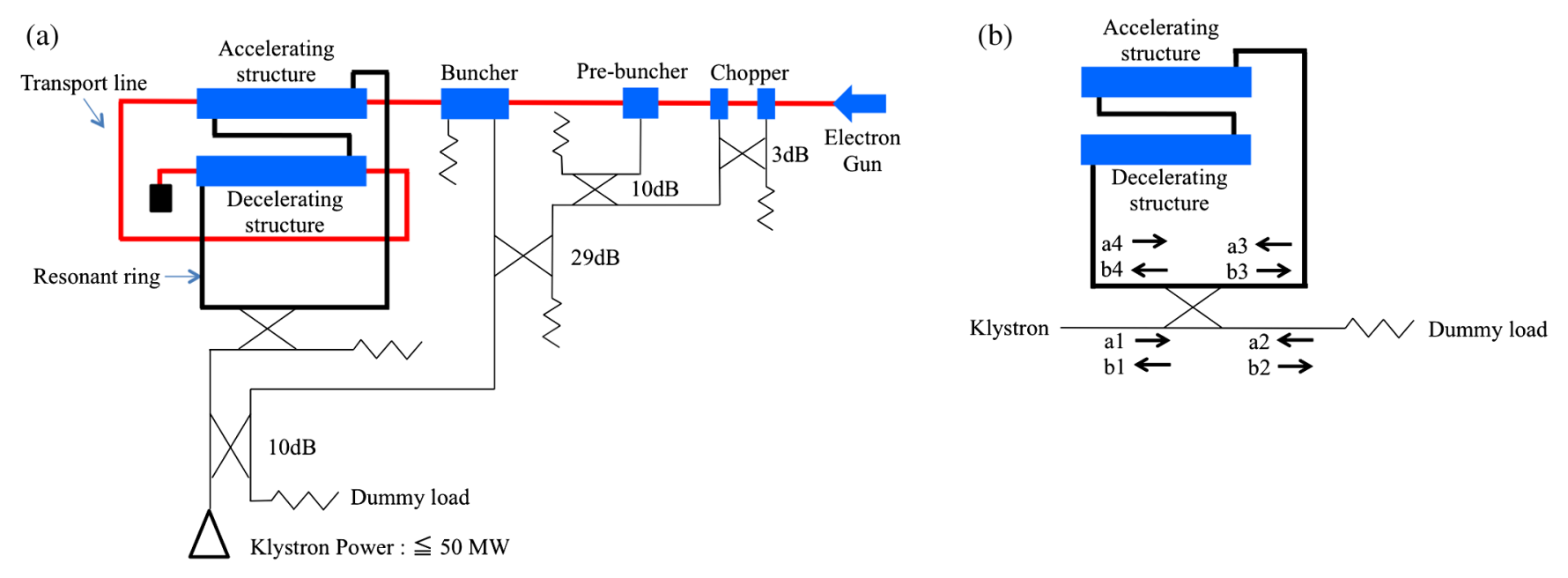

FIG. 3. (a) rf system of the PXR source and (b) the resonant ring. 
where $C$ is the voltage coupling coefficient of a directional coupler [31]:

$$
\begin{aligned}
\left(\begin{array}{l}
b_{1} \\
b_{2} \\
b_{3} \\
b_{4}
\end{array}\right) & =S_{i j}\left(\begin{array}{l}
a_{1} \\
a_{2} \\
a_{3} \\
a_{4}
\end{array}\right) . \\
S_{i j} & =\left(\begin{array}{cccc}
0 & \sqrt{1-C^{2}} & j C & 0 \\
\sqrt{1-C^{2}} & 0 & 0 & j C \\
j C & 0 & 0 & \sqrt{1-C^{2}} \\
0 & j C & \sqrt{1-C^{2}} & 0
\end{array}\right)
\end{aligned}
$$

The $a_{4}$ and $b_{3}$ are related as follows

$$
a_{4} \approx b_{3}(1-\tau)=b_{3} T,
$$

where $\tau$ and $T$ are the attenuation constant and the voltage transmission constant of the resonant ring. The field multiplication factor $M$ after $n$ cycles of the rf power into the resonant ring is given by

$$
\begin{aligned}
M\left(=\frac{b_{3}}{a_{1}}\right)= & j C\left\{1+T \sqrt{1-C^{2}}\right. \\
& \left.+\cdots+\left(T \sqrt{1-C^{2}}\right)^{n-1}\right\} \\
= & j C \frac{1-\left(T \sqrt{1-C^{2}}\right)^{n}}{1-T \sqrt{1-C^{2}}} .
\end{aligned}
$$

Also, the time evolution of the $b_{3}$ is written as

$b_{3}=\frac{C a_{1}}{1-T \sqrt{1-C^{2}}}\left\{1-\exp \left(\frac{1-T \sqrt{1-C^{2}}}{t_{f}} t\right)\right\}$,

where $t_{f}$ is the filling time. Table II summarizes the parameters specified for the resonant ring. Figure 4 shows the energy gain in the resonant ring as a function of the time for different coupling coefficients of the directional couplers. Figure 4 indicates that the coupling coefficient of the directional coupler should be $2 \mathrm{~dB}$ to achieve the beam energy of $72 \mathrm{MeV}$. The flattop of the rf pulse in this case is sufficiently long for our purpose.

TABLE II. Parameters of the resonant ring.

\begin{tabular}{lcc}
\hline \hline Parameters & Values & Units \\
\hline Input rf power & 45 & MW \\
Attenuation parameters & 0.905 & \\
Filling time & 200 & ns \\
\hline \hline
\end{tabular}

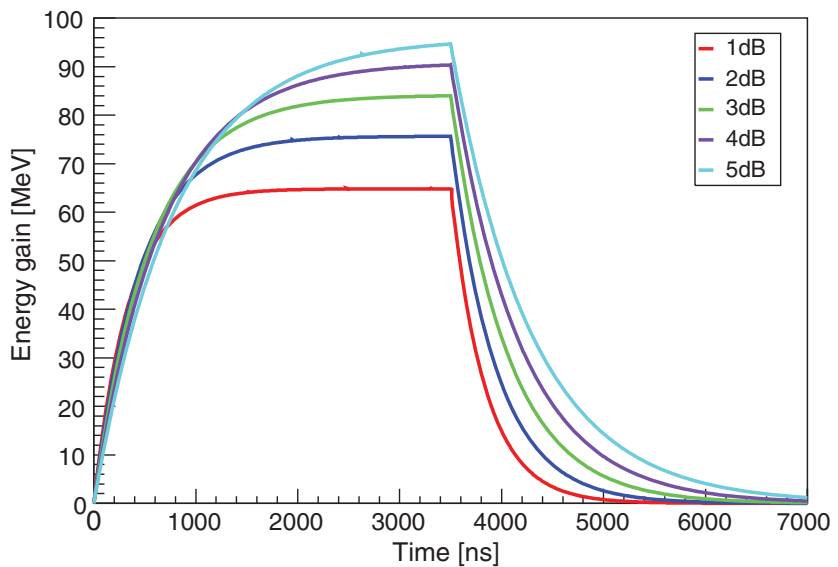

FIG. 4. Time dependence of the energy gain in the resonant ring for different coupling constants.

\section{EXPECTED PARAMETRIC X-RAY RADIATION}

The PXR is produced when electrons travel through a single crystal while they satisfy the Bragg condition, as illustrated in Figure 5. From the standpoint of quantum mechanics, the PXR is interpreted as diffraction of virtual photons associated with relativistic electrons by the crystal planes. A notable feature of the PXR is that its energy can be tuned by rotating the target crystal with respect to the incident electron beam. The energy of the PXR is given by $[32,33]$

$$
E=\hbar c \frac{g \sin \theta}{1-\cos \Omega} \stackrel{\Omega=2 \theta}{\longrightarrow} E=\hbar c \frac{g}{2 \sin \theta},
$$

where $\hbar$ is the Planck constant, $c$ is the velocity of light, $g$ is the reciprocal lattice of scattering planes. The reciprocal lattice $g$ for the diamond structure is

$$
g=\frac{2 \pi}{d}=\frac{2 \pi \sqrt{h^{2}+h^{2}+l^{2}}}{a}
$$

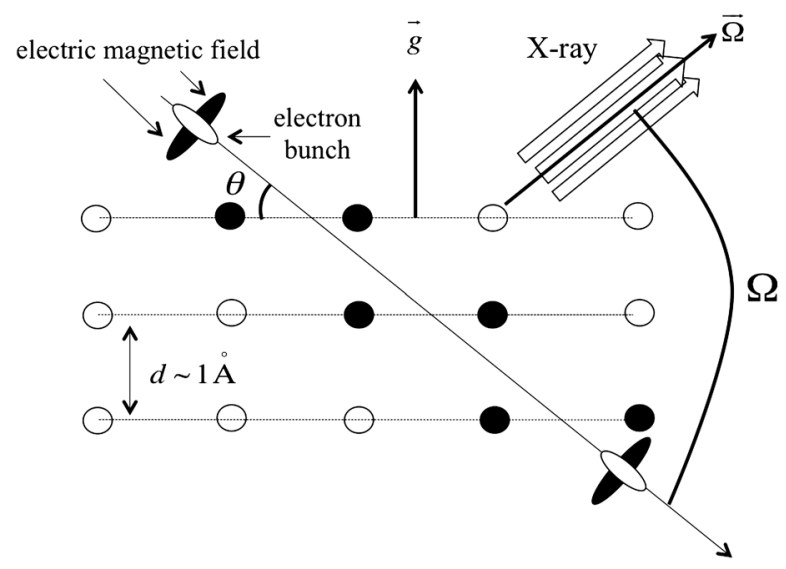

FIG. 5. Drawing of PXR generation. 
TABLE III. Basic parameters of diamond and Si crystals.

\begin{tabular}{lcccc}
\hline \hline Crystal & $\begin{array}{c}\text { Atomic } \\
\text { number }\end{array}$ & $\begin{array}{c}\text { Lattice } \\
\text { constant } \\
{[\AA]}\end{array}$ & $\begin{array}{c}\text { Plasma } \\
\text { frequency } \\
{[\mathrm{eV}]}\end{array}$ & $\begin{array}{c}\text { Debye } \\
\text { Temperature } \\
{[\mathrm{K}]}\end{array}$ \\
\hline Diamond & 6 & 3.567 & 38 & 1860 \\
$\mathrm{Si}$ & 14 & 5.431 & 31 & 625 \\
\hline \hline
\end{tabular}

where $a$ is the lattice constant, $d$ is the lattice spacing and $h$, $k, l$ are the Miller indices. Since the energy of PXR depends on the lattice spacing, a crystal with small lattice spacing $d$ and high Miller index $(h k l)$ is appropriate for producing high energy x-rays. In our case, diamond and Si crystals with the Miller index (111) are used in the simulation, since they have high Debye temperatures compared with other single crystals and it is easy to fabricate nearly perfect crystals. Table III shows the parameters of $\mathrm{Si}$ and diamond crystals. Diamond is better than $\mathrm{Si}$ for generating high energy x-rays due to its smaller lattice spacing. For rotating the Bragg angle to change the PXR energy, a PXR chamber as schematically shown by Fig. 6 will be used. It has three ports which cover the angular ranges of 6, 16-82 and 98-164 degrees. The energy ranges for the two crystals are summarized in Table IV.

The angular distribution of the PXR photons as observed outside the crystal per incident electron per steradian is given by [34]

$$
\begin{aligned}
& \frac{\partial^{2} N}{\partial \theta_{x} \partial \theta_{y}}= \frac{\alpha}{4 \pi c} \omega_{B}\left[L_{a}\left|\frac{\vec{g} \cdot \vec{\Omega}}{\vec{g} \cdot \vec{V}}\right|\left(1-\mathrm{e}^{\frac{-t}{L_{a} \mid \vec{g} \cdot \vec{\Omega}}}\right)\right] \mathrm{e}^{-2 W} \\
& \times \frac{|\chi|^{2}}{\sin ^{2} \theta_{B}} \frac{\theta_{x}^{2} \cos ^{2} 2 \theta_{B}+\theta_{y}^{2}}{\left(\theta_{x}^{2}+\theta_{y}^{2}+\theta_{p h}^{2}\right)^{2}} \\
& \theta_{p h}^{2}=\frac{1}{\gamma^{2}}
\end{aligned}
$$

where $\alpha$ is the fine structure constant, $\omega_{B}$ the $\mathrm{x}$-ray frequency, $c$ velocity of light, $L_{a}$ the x-ray absorption length, $t$ crystal thickness, $\theta_{B}$ the Bragg angle, $\chi$ the Fourier expansion of electric susceptibility, $\omega_{p}$ the plasma

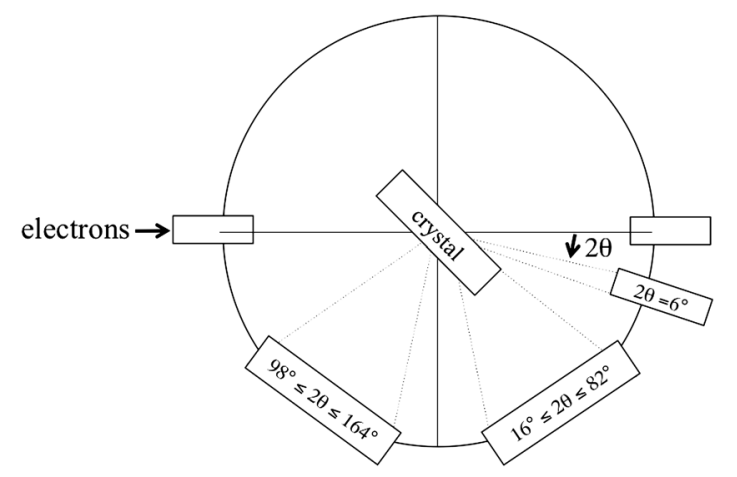

FIG. 6. Schematic of the PXR chamber.
TABLE IV. PXR energy dependence of theta for diamond and Si crystals with the Miller index (111).

\begin{tabular}{lccc}
\hline \hline $\begin{array}{l}\text { Observation } \\
\text { angle }\end{array}$ & $2 \theta=6^{\circ}$ & $16^{\circ}<2 \theta<82^{\circ}$ & $98^{\circ}<2 \theta<164^{\circ}$ \\
\hline Diamond & $67.5 \mathrm{keV}$ & $4.6 \mathrm{keV}-21.6 \mathrm{keV}$ & $3.0 \mathrm{keV}-4.0 \mathrm{keV}$ \\
$\mathrm{Si}$ & $37.8 \mathrm{keV}$ & $3.0 \mathrm{keV}-14.2 \mathrm{keV}$ & $2.0 \mathrm{keV}-2.6 \mathrm{keV}$ \\
\hline \hline
\end{tabular}

frequency, and $\theta_{x}$ and $\theta_{y}$ are the photon angle relative to the Bragg angle in the diffraction plane and the plane perpendicular to it, respectively. The $\mathrm{e}^{-2 W}$ is the DebyeWaller factor, which accounts for the thermal vibration of the lattice. It is approximately one at room temperature. The Fourier expansion of magnetic susceptibility $\chi$, which depends on the $\mathrm{x}$-ray energy, is written as

$$
\begin{gathered}
\chi(g)=-\frac{\lambda^{2} r_{e}}{\pi V_{c}} F(g), \\
F(g)=f(g) \sum_{j=1}^{n} \exp \left\{-2 \pi\left(h x_{j}+k y_{j}+l z_{j}\right)\right\}, \\
f(g)=f_{0}(g)+f^{\prime}(g)+i f^{\prime \prime}(g),
\end{gathered}
$$

where $r_{e}$ is the classical electron radius, $\lambda$ the wavelength of PXR, $V_{c}$ the volume of the unit cell, $F_{0}$ the crystal structure factor and $\left(f_{0}, f^{\prime}, f^{\prime \prime}\right)$ the atomic form factors. In general, $f^{\prime}$ and $f^{\prime \prime}$ can be ignored except in the vicinity of the absorption edge energy (around $2 \mathrm{keV}$ for $\mathrm{Si}$ ). Since $F$ depends on the crystal Miller indices and $g, \chi$ is generally of the order of $10^{-5}-10^{-6}$ for most crystals [13]. This small value of $\chi$ is the main reason for low PXR yields. Figure 7 shows the dependence of $\chi$ on the $\mathrm{x}$-ray energy for Si and diamond crystals with the Miller index (111) by using Eq. (15). The photon yield also depends on the factor of $1 / \sin ^{2} \theta_{B}$, which means that the reciprocal lattice

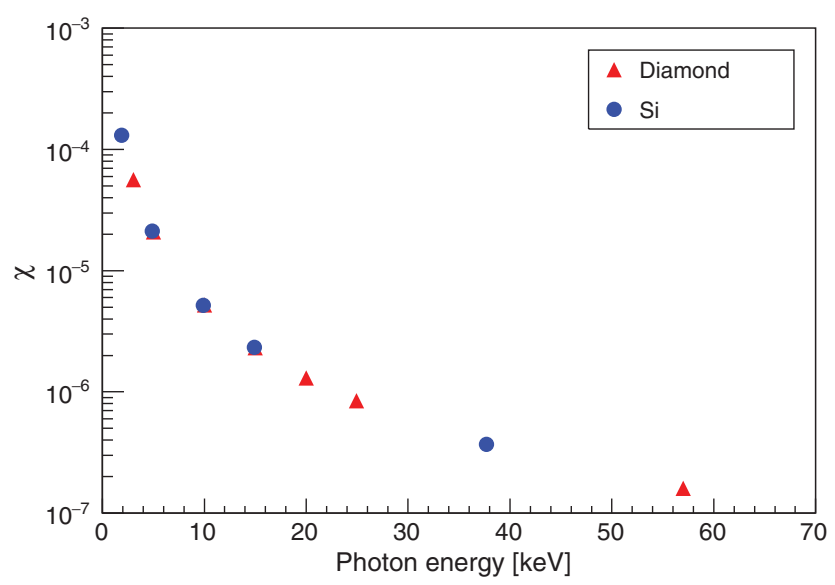

FIG. 7. The dependence of $\chi$ on x-ray energy for diamond and Si with the Miller index (111). 


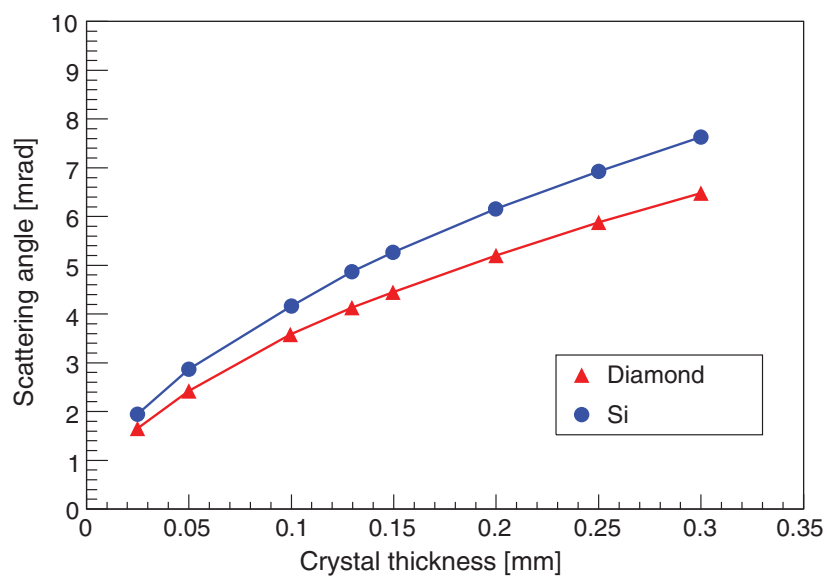

FIG. 8. Scattering angle estimated from fitting a Gaussian function depending on crystal thickness of $\mathrm{Si}$ and diamond. The beam tail is ignored.

constant $g$ from Eqs. (11) and (12) should be large for high photon yield. Therefore, we choose the Miller index (111) rather than (100) and (110) for Si and diamond crystals.

The electrons are deflected due to Coulomb scattering from nuclei when they pass through the crystal. As a consequence, angular broadening of the PXR and emittance growth of the electron beam occur. The multiple scattering in the crystal can be simulated with GEANT4. Figure 8 shows the scattering angle of electrons with the energy of $75 \mathrm{MeV}$ after passing through diamond and $\mathrm{Si}$ crystals as a function of the thickness. For reducing particle loss and the thickness of the radiation shield, a thin crystal is preferred for the target. We have chosen the crystal thickness of $0.1 \mathrm{~mm}$ and $0.13 \mathrm{~mm}$ for $\mathrm{Si}$ and diamond, respectively, so that the emittances after the crystal are approximately $60 \mathrm{~mm}$-mrad. More on the beam loss will be discussed in Sec. V.

The rms scattering angle after traversing the thickness $\mathrm{L}$ is expressed as [35]

$$
\begin{gathered}
\theta=\frac{13.6[\mathrm{MeV}]}{\beta c p} \sqrt{\frac{L}{L_{\mathrm{rad}}}}\left[1+0.038 \ln \left(\frac{L}{L_{\mathrm{rad}}}\right)\right], \\
L_{\mathrm{rad}}=\frac{716.4\left[\mathrm{~g} / \mathrm{cm}^{2}\right] \cdot A}{Z(Z+1) \ln (287 / \sqrt{z})},
\end{gathered}
$$

where $\beta c$ is the velocity of the electrons, $p$ the momentum of the electrons, $Z$ the atomic number, $A$ the mass number and $L_{\mathrm{rad}}$ the radiation length of the crystal which is given by Eq. (19) [36]. For a Si crystal with the thickness of $0.1 \mathrm{~mm}$, eq. (18) gives $\theta \approx 4.3 \mathrm{mrad}$, and it is comparable with the GEANT4 simulation result of $\theta=4.1 \mathrm{mrad}$. The angular broadening of the PXR caused by multiple Coulomb scattering can be estimated by calculating the convolution of the Gaussian distribution of multiple scattering with the PXR yield in Eq. (13). Figure 9 shows the number of PXR

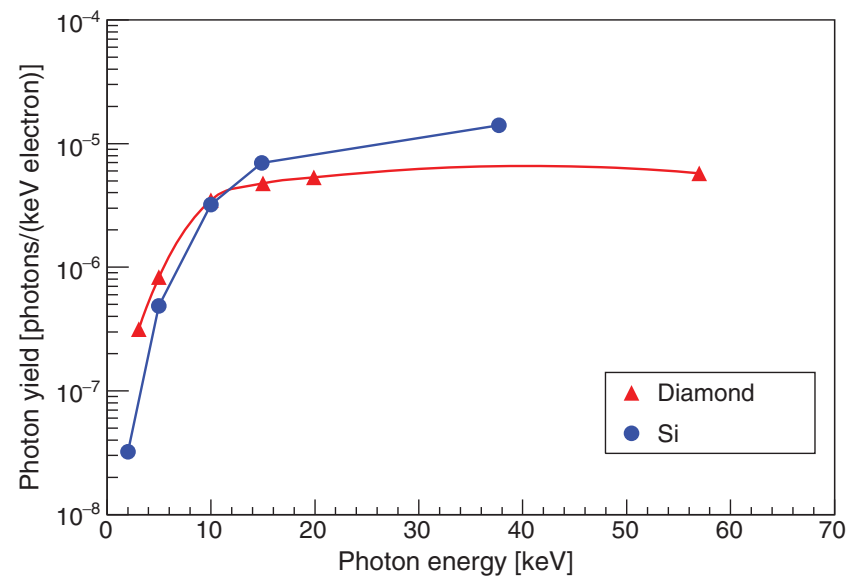

FIG. 9. The dependence of PXR yield on the X-ray energy for $\mathrm{Si}$ and diamond with the Miller index (111).

photons expected to be detected per electron. Here, the effects of the crystals thickness of $0.1 \mathrm{~mm}$ and $0.13 \mathrm{~mm}$ are included, and an x-ray detector with an aperture of $3.0 \mathrm{~cm}^{2}$ is assumed to be located at $1 \mathrm{~m}$ from the crystal in the Bragg geometry. The rms beam size at the crystal is assumed to be $0.1 \mathrm{~mm}$, sufficiently small for preventing thermal destruction of the crystal and for maintaining the beam size to fit within the vacuum chamber after multiple scattering in the crystal. Figure 9 shows that for the PXR yield to be more than $1 \times 10^{9}$ photons $/ \mathrm{s}$ at $15 \mathrm{keV}$, the average beam current must be $25 \mu \mathrm{A}$ and $34 \mu \mathrm{A}$ for $\mathrm{Si}$ and diamond, respectively.

LEBRA has experienced that the destruction of a $\mathrm{Si}$ crystal was caused due to crystal heating during one micropulse, and that it did not depend on the average beam current [16]. We estimated the maximum crystal temperature during a micro-pulse of $2 \mu \mathrm{s}$ with a finite element method. Assuming that the energy loss of an electron beam

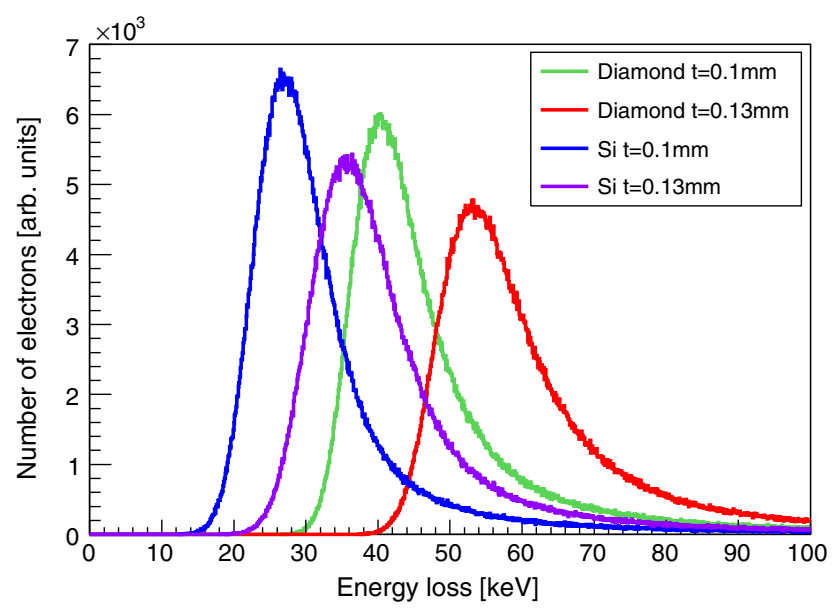

FIG. 10. Electron's energy loss after the electron beam of $75 \mathrm{MeV}$ passes through $\mathrm{Si}$ and diamond crystals of different thickness. Symbol t in the legend means crystal thickness. 


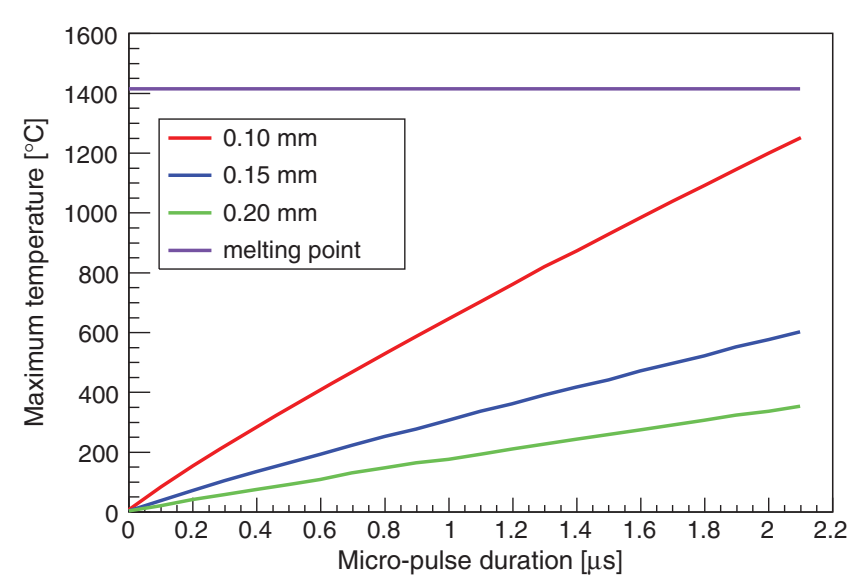

FIG. 11. Temperature change of the $0.1 \mathrm{~mm}$ thick Si crystal during a micro-pulse duration of $2 \mu$ s for different beam sizes at the crystal.

in a crystal is all converted into thermal energy, we first estimated it to be caused by the ionization loss and bremsstrahlung using GEANT4. Figure 10 shows the energy loss of the beam after the electron beam of $75 \mathrm{MeV}$ passes through diamond and $\mathrm{Si}$ crystals with different thickness. It shows for example, that the energy loss in a $0.1 \mathrm{~mm}$ thick $\mathrm{Si}$ crystal peaks at $26 \mathrm{keV}$. Then, the thermal load for a peak current of $300 \mathrm{~mA}$ (maximum current of the electron gun) can be computed to be $7.8 \mathrm{~kW}(\approx 26 \mathrm{keV} \times 0.3 \mathrm{~A})$ for the $0.1 \mathrm{~mm}$ thick $\mathrm{Si}$ crystal. Using this thermal load, we simulated the crystal temperature for different electron beam sizes at the $\mathrm{Si}$ crystal, and the result is shown in Fig. 11. Since Si has a melting point of $1414{ }^{\circ} \mathrm{C}$, the $\mathrm{Si}$ crystal with the thickness of $0.1 \mathrm{~mm}$ should not be destroyed when the peak current is about $300 \mathrm{~mA}$.

\section{OPTICS FOR BEAM TRANSPORT}

An important requirement from the standpoint of beam optics is to minimize beam losses to achieve low radiation level. When high-energy electrons hit matter, many photons of a wide energy range are generated. To study the number of neutrons and photons and their energies generated due to beam losses, we used GEANT4 to simulate the interaction of $10^{7}$ electrons with an energy of $75 \mathrm{MeV}$ with stainless steel ( $2.5 \mathrm{~mm}$ thick for the beam pipe), copper (10 $\mathrm{mm}$ thick for the decelerating structure), and graphite (200 mm thick for the beam dump). The simulation results are shown in Figs. 12 and 13. A sharp peak at $0.511 \mathrm{MeV}$ in Fig. 12 can be interpreted as the effect of pair annihilation which occurs by collision of electrons and positrons. Moreover, Figs. 12 and 13 indicate that the particle loss in the decelerating structure should be kept as low as possible, because a large number of gamma rays with a peak at about $0.2 \mathrm{MeV}$ and neutrons are produced. On the other hand, for the particle loss in beam line of the stainless steel, gamma rays with $70 \mathrm{KeV}$ energy peak are generated, while the number of

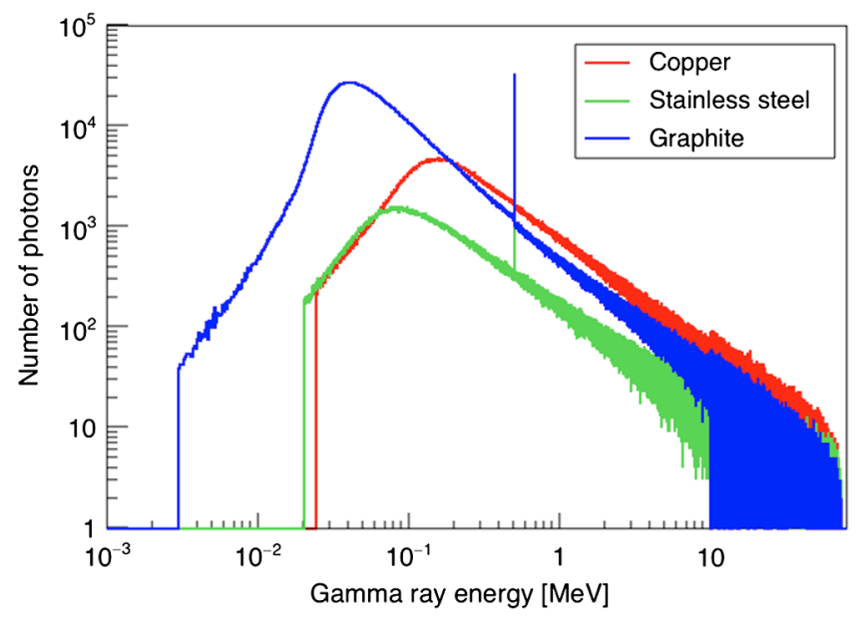

FIG. 12. Gamma rays generated when a beam with $10^{7}$ electrons at $75 \mathrm{MeV}$ collides with copper, stainless steal and graphite.

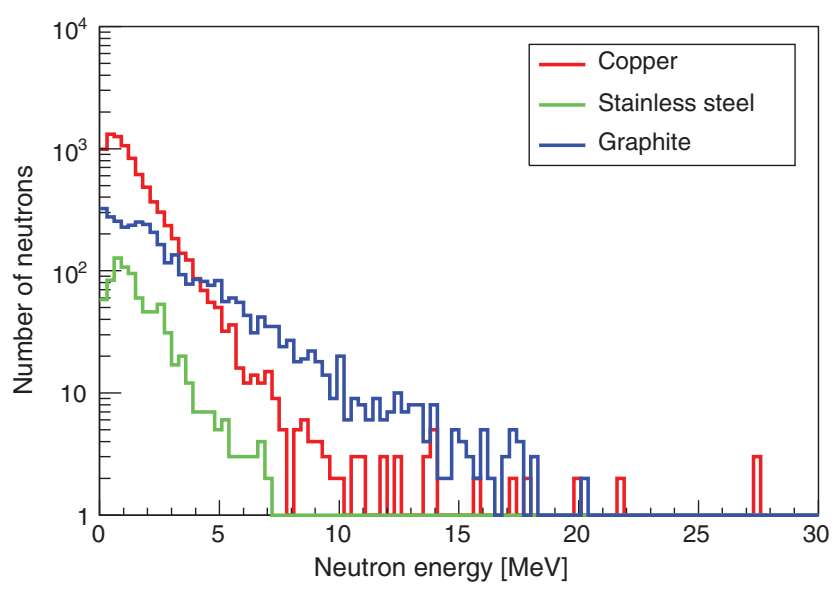

FIG. 13. Neutrons generated when a beam with $10^{7}$ electrons at $75 \mathrm{MeV}$ electrons collides with copper, stainless steal and graphite.

neutrons is relatively small although they are produced. If the electron beam of $75 \mathrm{MeV}$ is dumped at the beam dump consisting of graphite, a lot of gamma rays and neutrons with wide energy ranges are emitted. Therefore, to suppress the generation of these unwanted particles, we dump the electron beam after the electron energy is decreased.

The beam transport line must be designed with considerations on the emittance growth and energy loss of the beam after electrons pass through the radiator crystal. Three points should be noted: (1) rms beam sizes should be less than $2.6 \mathrm{~mm}$ in the transport line, $0.1 \mathrm{~mm}$ at the target crystal and $1.3-1.6 \mathrm{~mm}$ in the accelerating and decelerating structures, (2) energy spread should be below $\pm 0.25 \%$ and (3) the length of quadrupole magnets is 45,70 or $90 \mathrm{~mm}$, and their field strengths should be in the range of 5-20 $\mathrm{T} / \mathrm{m}$. Table $\mathrm{V}$ tabulates the assumed beam parameters at the buncher exit. Using SAD [23], we calculated the beam 
TABLE V. Beam parameters at the buncher exit.

\begin{tabular}{lcc}
\hline \hline Parameters & Values & Units \\
\hline Beam energy & 3 & $\mathrm{MeV}$ \\
Normalized emittance & 20 & $\mathrm{~mm}-\mathrm{mrad}$ \\
Bunch length & 1 & $\mathrm{~mm}$ \\
$\alpha_{x}=\alpha_{y}$ & 0 & \\
$\beta_{x}=\beta_{y}$ & 0.3 & $\mathrm{~m}$ \\
$\Delta E / E$ & \pm 0.15 & $\%$ \\
\hline \hline
\end{tabular}

optics except at the beam dump with the initial parameters from the buncher exit to the decelerating structure. Figure 14 shows the beam optics from the buncher exit to the decelerating structure along the path length when the interactions of the beam with the radiator crystal are ignored. Also, Figure 15 shows the beam optics from the collision point to the decelerating structure when a $\mathrm{Si}$ crystal with the thickness of $0.1 \mathrm{~mm}$ is inserted and when it is not. The details on the initial parameters after the crystal will be discussed later. The plots (a), (b), and (c) in Figs. 14 and 15 indicate the beta functions, the dispersion, and the rms beam sizes. The red arrows show the collision point, where the beam size is $\sim 0.1 \mathrm{~mm}$, and the dispersion is zero except in the arc sections. The electron beam can be transported from the exit of the decelerating structure when the crystal is not inserted. However, when the crystal is inserted, the vertical beam size reaches its maximum at a distance of $1 \mathrm{~m}$ after the collision point. Therefore, a quadrupole magnet with a sufficiently large bore diameter $(50 \mathrm{~mm})$ will be used. If a beam pipe with a diameter of $26 \mathrm{~mm}$ is used, the number of neutrons produced there is estimated to be about $10^{7}$ for an electron beam of $35 \mu \mathrm{A}$. A thick shielding wall around it should be used for this part.

The bunch length was simulated by particles tracking with SAD, and then the energy loss was ignored. For the

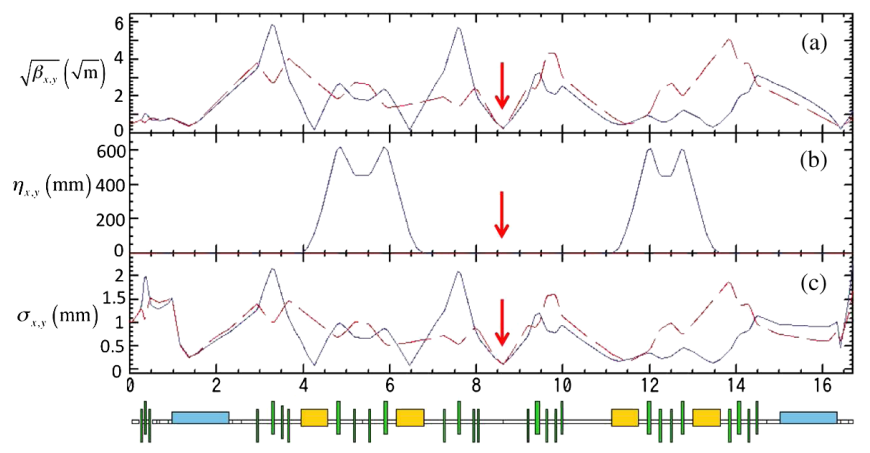

FIG. 14. Beam optics from the exit of the buncher to the exit of the decelerating structure, when interactions of the beam with the target (location indicated by red arrows) are ignored. (a) $\sqrt{\beta}$ function, (b) dispersion function and (c) beam size. Blue and red lines show the horizontal and vertical planes. Blue boxes are accelerating and decelerating structures, green boxes are quadrupole magnets, and yellow boxes are bending magnets.
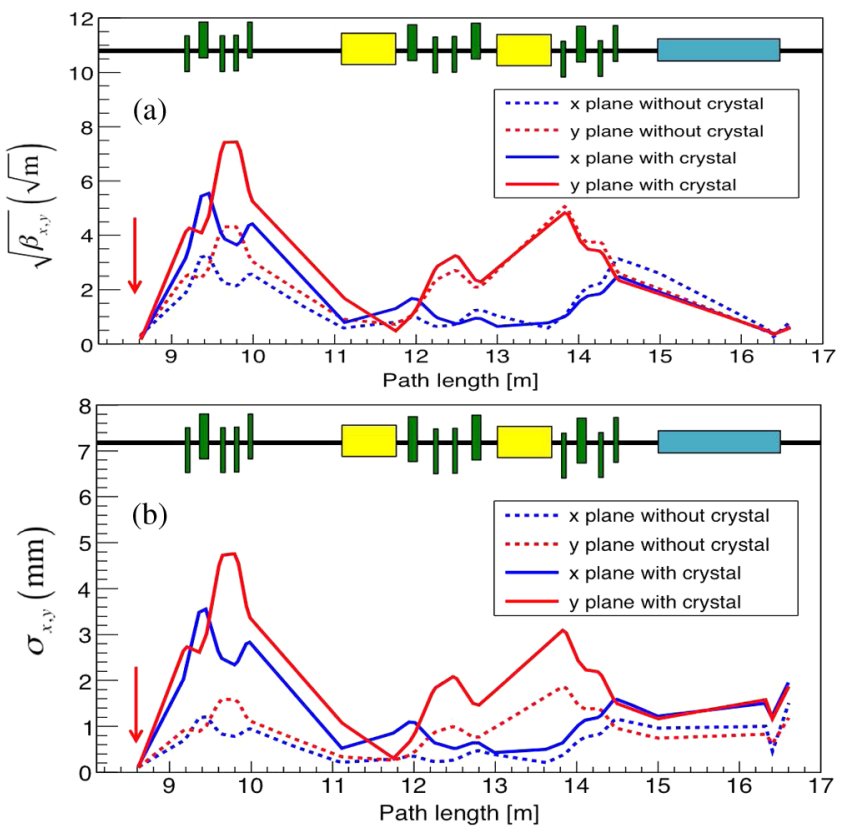

FIG. 15. Beam optics from the collision point to the decelerating structure along the path length, when the target (location indicated by a red arrow) is inserted (lines) and not inserted (dotted lines). (a) $\sqrt{\beta}$ function and (b) beam size. Blue and red lines show the horizontal and vertical planes. Blue boxes are decelerating structure, green boxes are quadrupole magnets, and yellow boxes are bending magnets.

thickness of $0.1 \mathrm{~mm}(\mathrm{Si})$ and $0.13 \mathrm{~mm}$ (diamond), electron energy loss is less than $60 \mathrm{keV}$ from Fig. 10, which is $0.08 \%$ of the incident energy of $75 \mathrm{MeV}$. This is low enough to be ignored. Figure 16 shows the longitudinal particle distribution at the buncher exit, the collision point and the decelerating structure. The total bunch length at each place is about $0.8 \mathrm{~mm}, 1.3 \mathrm{~mm}$, and $2 \mathrm{~mm}$, respectively. Since the bunch length at the decelerating structure is $2 \mathrm{~mm}$, the electrons energy can be reduced to $7 \mathrm{MeV}$ or lower, when the electron bunch is on the decelerating phase

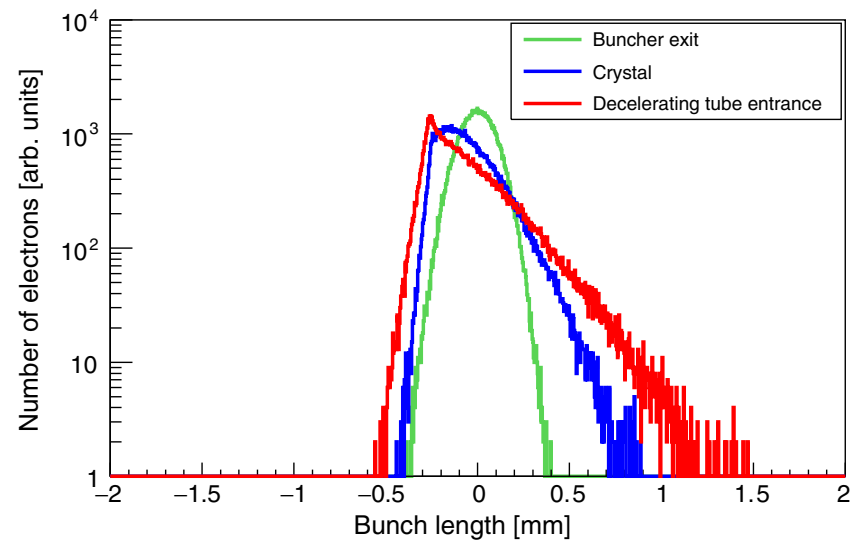

FIG. 16. Bunch length at the exit of the buncher (green), the collision point (blue), and the decelerating structure (red). 
of the rf wave. To maintain this condition in operation we control the path length of the transport line by moving the second arc section on the movable stage.

The emittance and energy spread after electrons travel through the crystal are evaluated as follows. From Fig. 8, the electrons after passing through the $\mathrm{Si}$ and diamond crystals with the thickness of $0.1 \mathrm{~mm}$ and $0.13 \mathrm{~mm}$ have the scattering angle of about $4.1 \mathrm{mrad}$ for the Gaussian fit. This result does not take into account the effect of the beam divergence of $1.36 \mathrm{mrad}$ at the crystal surface. The final beam divergence including the initial beam divergence can be computed to be about $4.3 \mathrm{mrad}\left(=\sqrt{4.1^{2}+1.36^{2}}\right)$. The standard deviation for the entire distribution including the beam tails is $20-25 \%$ larger than that for the Gaussian fit. We employed the scattering angle estimated by the Gaussian fit, because the number of particles in the tail part is small. Then, the normalized emittances can be computed approximately using the beam size $(\sigma)$ and beam divergence $(\theta)$ at the exit of crystal,

$$
\begin{aligned}
\varepsilon_{x, y} & =\sigma \cdot \theta \cdot(\gamma \beta)=0.1 \mathrm{~mm} \times(4.1-4.3) \mathrm{mrad} \times \frac{75}{0.511} \\
& \approx 60-63 \mathrm{~mm} \cdot \mathrm{mrad} .
\end{aligned}
$$

After electrons pass through the crystal, the beam sizes and $\alpha_{x, y}$ are approximately conserved while the $\beta_{x, y}$ and emittances change. In this emittance computation, planar channeling and rainbow scattering [37] in the crystal are not taken into account. Because the beam is strongly focused at the crystal of sizes close to $0.1 \mathrm{~mm}$, the beam divergences are large enough to ignore these physical phenomena. We used the normalized emittance of $60 \mathrm{~mm}$-mrad when the beam optics was simulated since the effect of this narrow emittance range on beam optics is very small. Also, the beam energy spread at the front of the crystal is about $1.55 \times 10^{-4}$, and the energy spread after the diamond crystal about $3.35 \times 10^{-4}$. With this emittance and energy spread, the beam optics in Fig. 15 was calculated.

\section{CONCLUSIONS}

In this paper we discussed a proposal of a small size $(4 \mathrm{~m} \times 8 \mathrm{~m})$ and high intensity PXR source based on a linear accelerator resembling an energy recovery linac, and have presented a complete, workable design of a PXR test facility. This x-ray source, whose beam energy is $75 \mathrm{MeV}$, is a relatively compact $\mathrm{x}$-ray source compared to typical $100 \mathrm{MeV}$-class accelerators. The target value for the PXR flux, over $10^{9}$ photons/s with energies higher than $15 \mathrm{keV}$, can be achieved with an average beam current of $25 \mu \mathrm{A}$ with a $\mathrm{Si}(0.1 \mathrm{~mm}$ thick) target and $34 \mu \mathrm{A}$ with a diamond (0.13 mm thick) with the Miller index (111) in the Bragg geometry. The x-rays produced can cover a wide energy range from $2 \mathrm{keV}$ to $57.5 \mathrm{keV}$. The crystal thickness is chosen so that the electron beam emittance is $60 \mathrm{~mm}$-mrad after it passes through the crystal so as to keep the beam loss under control in the high-energy transport line.

To realize the low radiation level at the beam dump, we dump the electron beam after beam energy is decreased below $7 \mathrm{MeV}$ with the decelerating structure. This results in practically no neutron emission. Accelerating and decelerating structures, are made of copper with $6 \mathrm{~N} 8$ grade, and are operated at $20 \mathrm{~K}$ to operate with long beam pulses. The Q-factor of the accelerating structures at $20 \mathrm{~K}$ would be about 50000 . They are operated in the resonant ring configuration to achieve an accelerating gradient of $55 \mathrm{MeV} / \mathrm{m}$.

The beam transport line after the accelerating structure was designed with SAD. The beam optics ensures that the dispersion is zero except in the two arc sections. The rms beam sizes in the crystal, in the transport line and in the two structures are below $0.1 \mathrm{~mm}, 2.6 \mathrm{~mm}$ and $13-16 \mathrm{~mm}$, respectively. Transporting electrons to the beam dump is easy if no loss occurs due to the crystals. On the other hand, substantial multiple scattering and particle loss occur when the crystal is inserted. Therefore, thick concrete wall and quadrupole magnets with large bore diameter are required to suppress particle losses and their impact as much as possible. Also, the longitudinal bunch length was simulated by particle tracking. The bunch length at the decelerating structure entrance is about $2 \mathrm{~mm}$, and the electron bunch energy can be decreased to less than $7 \mathrm{MeV}$.

\section{ACKNOWLEDGMENTS}

We would like to thank Dr. N. Toge (KEK), Dr. T. Suwada (KEK), and Dr. T. Sen (FNAL) for useful discussions and suggestions.

[1] P. Duke, Synchrotron Radiation: Production and Properties (Oxford University Press, New York, 2008), Vol. 3.

[2] J. A. Bearden, X-ray wavelengths, Rev. Mod. Phys. 39, 78 (1967).

[3] M. O. Krause and J. H. Oliver, Natural widths of atomic K and $L$ levels, $K \alpha \mathrm{X}$-ray lines and several $K L L$ Auger lines, J. Phys. Chem. Ref. Data 8, 329 (1979).

[4] M. A. Piestrup, Xizeng Wu, V. V. Kaplan, S. R. Uglov, J. T. Cremer, D. W. Rule, and R. B. Fiorito, A design of mammography units using a quasimonochromatic $\mathrm{X}$-ray source, Rev. Sci. Instrum. 72, 2159 (2001).

[5] V. G. Baryshevsky, V. A. Danilov, O. L. Ermakovich, I. D. Feranchuk, A. V. Ivashin, V. I. Kozus, and S. G. Vinogradov, Angular distribution of parametric X-rays, Phys. Lett. 110A, 477 (1985).

[6] J. Freudenberger, V. B. Gavrikov, M. Galemann, H. Genz, L. Groening, V. L. Morokhovskii, V. V. Morokhovskii, U. Nething, A. Richter, J. P. F. Sellschop, and N. F. Shul'ga, Parametric X-Ray Radiation Observed in Diamond at Low Electron Energies, Phys. Rev. Lett. 74, 2487 (1995).

[7] J. Freudenberger, H. Genz, V. V. Morokhovskii, A. Richter, V. L. Morokhovskii, U. Nething, R. Zahn, and J.P. F. Sellschop, Lineshape, linewidth and spectral density of 
parametric x-radiation at low electron energy in diamond, Appl. Phys. Lett. 70, 267 (1997).

[8] T. Sen and C. Lynn, Spectral brilliance of channeling radiation at the ASTA photoinjector, Int. J. Mod. Phys. A 29, 1450179 (2014).

[9] W. Wagner, B. Azadegan, M. Sobiella, J. Steiner, K. Zeil, and J. Pawelke, An intense channeling radiation source, Nucl. Instrum. Methods Phys. Res., Sect. B 266, 327 (2008).

[10] J. Hyun, D. Broemmelsiek, D. Edstrom, T. Sen, D. Mihalcea, P. Piot, and W. Rush, Expected results from channeling radiation experiments at fast, in Proceedings of the 7th International Particle Accelerator Conference (IPAC'16), Busan, Korea (JACOW, Geneva, Switzerland, 2016), p. 1873.

[11] L. Wartski, S. Roland, J. Lasalle, M. Bolore, and G. Filippi, Interference phenomenon in optical transition radiation and its application to particle beam diagnostics and multiple scattering measurements, J. Appl. Phys. 46, 3644 (1975).

[12] S. Asano, I. Endo, M. Harada, S. Ishii, T. Kobayashi, and T. Nagata, How Intense is Parametric X Radiation?, Phys. Rev. Lett. 70, 3247 (1993).

[13] B. Sones, Y. Danon, and R. C. Block, Lithium fluoride (LiF) crystal for parametric X-ray (PXR) production, Nucl. Instrum. Methods Phys. Res., Sect. B 227, 22 (2005).

[14] A. V. Schagin and N. A. Khizhnyak, Differential properties of parametric x-ray radiation from a thin crystal, Nucl. Instrum. Methods Phys. Res., Sect. B 119, 115 (1996).

[15] M. Inagaki, Y. Hayakawa, K. Nogami, T. Tanaka, K. Hayakawa, T. Sakai, K. Nakao, and I. Sato, Wavelength dispersive $\mathrm{x}$-ray absorption fine structure imaging by parametric x-ray radiation, Jpn. J. Appl. Phys. 47, 8081 (2008).

[16] Y. Hayakawa, Y. Takahashi, T. Kuwada, T. Sakae, T. Tanaka, K. Nakao, K. Nogami, M. Inagaki, K. Hayakawa, and I. Sato, X-ray imaging using a tunable coherent X-ray source based on parametric x-ray radiation, J. Inst. 8, C08001 (2013).

[17] Y. Hayakawa, I. Sato, K. Hayakawa, and T. Tanaka, Simulations to the project of a PXR based x-ray source composed of an electron linac and a double-crystal system, Nucl. Instrum. Methods Phys. Res., Sect. B 227, 32 (2005).

[18] Y. Hayakawa, I. Sato, K. Hayakawa, T. Tanaka, A. Mori, T. Kuwada, T. Sakai, K. Nogami, K. Nakao, and T. Sakae, Status of the parametric $\mathrm{x}$-ray generator at LEBRA, Nucl. Instrum. Methods Phys. Res., Sect. B 252, 102 (2006).

[19] Y. Hayakawa, K. Hayakawa, M. Inagaki, T. Kuwada, K. Nakao, K. Nogami, T. Sakai, I. Sato, Y. Takahashi, and T. Tanaka, Geometrical effect of target crystal on PXR generation as a coherent X-ray source, Int. J. Mod. Phys. A 25, 174 (2010).

[20] J. Guo, S. Tantawi, D. Martin, and C. Yoneda, Cryogenic rf material testing with a HighQ copper cavity, in AIP Conference Proceedings (American Institute of Physics, Annapolis, 2010), p. 330.
[21] D. Proch, Superconducting cavities for accelerators, Rep. Prog. Phys. 61, 431 (1998).

[22] http://geant4.cern.ch.

[23] http://acc-physics.kek.jp/SAD/.

[24] Y. L. Wang, T. Emoto, M. Nomura, S. Toyama, and I. Sato, A new chopper system for high power $\mathrm{CW}$ linac, in Proceedings of the 1994 International Linac Conference, Tsukuba, Japan (KEK, Tsukuba, Japan, 1994), p. 205.

[25] M. B. Chadwick, P. Oblozinsky, A. I. Blokhin, T. Fukahori, Y. Han, Y. O. Lee, M. N. Martins, S. F. Mughabghab, V. V. Varlamov, B. Yu et al., Report No. IAEA TECH-DOC 1178, 2000.

[26] G. E. H. Reuter and E. H. Sondheimer, The theory of the anomalous skin effect in metals, Proc. R. Soc. A 195, 336 (1948).

[27] W. Chou and F. Ruggiero, CERN Technical Report No. CERN-LHC-Project-Note-2, 1995.

[28] E. W. Johnson and H. H. Johnson, Frequency dependence of the anomalous skin effect in high-purity copper, J. Appl. Phys. 36, 1286 (1965).

[29] S. Inagaki, E. Ezura, J. F. Liu, and H. Nakanishi, Thermal expansion and microwave surface reactance of copper from the normal to anomalous skin effect region, J. Appl. Phys. 82, 5401 (1997).

[30] J. E. Jensen, W. A. Tuttle, R. B. Stewart, H. Brenchna, and A. G. Prodell, Brookhaven National Laboratory Selected Cryogenic Data Notebook: Sections X (Brookhaven National Laboratory, New York, 1980).

[31] E. Gerken, J. M. Gahl, D. Rees, W. Roybal, and K. Cummings, Proceedings of the Particle Accelerator Conference, Vancouver, BC, Canada, 1997 (IEEE, New York, 1997), Vol. 3, p. 3725.

[32] T. Akimoto, M. Tamura, J. Ikeda, Y. Aoki, F. Fujita, K. Sato, A. Honma, T. Sawamura, M. Narita, and K. Imai, Generation and use of parametric x-rays Generation and use of parametric x-rays with an electron linear accelerator, Nucl. Instrum. Methods Phys. Res., Sect. A 459, 78 (2001).

[33] T. Sen, in Proceedings of the 7th International Particle Accelerator Conference (IPAC'16), Busan, Korea (JACOW, Geneva, Switzerland, 2016). p. 1877.

[34] K. H. Brenzinger, C. Herberg, B. Limburg, H. Backe, S. Dambach, H. Euteneuer, F. Hagenbuck, H. Hartmann, K. Johann, K. H. Kaiser, O. Kettig, G. Knies, G. Kube, W. Lauth, H. Schope, and Th. Walcher, Investigation of the production mechanism of parametric x-ray radiation, $\mathrm{Z}$. Phys. A 358, 107 (1997).

[35] J. Beringer et al., Review of particle physics, Phys. Rev. D 86, 010001 (2012).

[36] D. E. Groom et al., Review of particle physics, Eur. Phys. J. C15, 1 (2000).

[37] S. P. Fomin and N.F. Shul'ga, Rainbow scattering and orbiting of fast particles in crystals, Phys. Lett. 73A, 131 (1979). 